\title{
Erratum to: Impact of Imitation Processes on the Effectiveness of Ring Vaccination
}

\author{
Chad R. Wells • Jean M. Tchuenche • \\ Lauren Ancel Meyers • Alison P. Galvani • \\ Chris T. Bauch
}

Published online: 16 October 2014

(C) Society for Mathematical Biology 2014

\section{Erratum to: Bull Math Biol (2011) 73:2748-2772 DOI 10.1007/s11538-011-9646-4}

In Section 2, under the scenario of case 2: where the vaccine may work in time to prevent infection, $(\lambda+\omega<\sigma+\delta)$, there was an error in the payoff to vaccinate (Eq. $6)$. The probability that the individual was not infected prior to the vaccine becoming effective was incorrect; the given probability was $(1-p)^{\lambda+\omega-\delta}$ when it should have been $(1-p)^{\lambda+\omega-\sigma}$. The correct payoff to vaccinate function is

$$
\begin{aligned}
P_{V}= & \left(L-r_{\mathrm{vac}}-r_{\mathrm{inf}}\right)\left\{\left[1-(1-p)^{\lambda+\omega-\sigma}\right]\right. \\
& \left.+\left[(1-\varepsilon)(1-p)^{\lambda+\omega-\sigma}\left(1-(1-p)^{\delta-(\lambda+\omega-\sigma)}\right)\right]\right\} \\
& +\left(L-r_{\mathrm{vac}}\right)\left\{\left[\varepsilon(1-p)^{\lambda+\omega-\sigma}\left(1-(1-p)^{\delta-(\lambda+\omega-\sigma)}\right)\right]+(1-p)^{\delta}\right\}
\end{aligned}
$$

The online version of the original article can be found under doi:10.1007/s11538-011-9646-4.

C. R. Wells $(\bowtie) \cdot$ J. M. Tchuenche · C. T. Bauch

Department of Mathematics and Statistics, University of Guelph, Guelph, Ontario, Canada e-mail: cwells01@uoguelph.ca

L. A. Meyers

Section of Integrative Biology, University of Texas, Austin, TX, USA

\section{A. P. Galvani}

School of Public Health, Yale University, New Haven, CT, USA

C. T. Bauch

Department of Epidemiology, Biostatistics, and Occupational Health,

McGill University, Montreal, Quebec, Canada 
Table 1 Parameter definitions and baseline values for the corrected version

\begin{tabular}{|c|c|c|}
\hline Parameter & Definition & Baseline value \\
\hline$Q$ & $\begin{array}{l}\text { Number of contacts of the index } \\
\text { case }\end{array}$ & 10 or 100 \\
\hline$p$ & $\begin{array}{l}\text { Transmission probability per } \\
\text { edge per day }\end{array}$ & 0.05 \\
\hline$\omega$ & Incubation period & 5 days \\
\hline$\sigma$ & Latent period & 4 days \\
\hline$\delta$ & Infectious period & 5 days \\
\hline$\lambda$ & $\begin{array}{l}\text { Time between decision to } \\
\text { vaccinate and attainment of } \\
\text { protective immunity }\end{array}$ & 1 day \\
\hline$\varepsilon$ & Vaccine efficacy & 0.95 \\
\hline$r_{\mathrm{vac}}$ & $\begin{array}{l}\text { Penalty due to being vaccinated } \\
\text { (e.g. adverse events, potential } \\
\text { monetary cost) }\end{array}$ & 0.024 \\
\hline$r_{\text {inf }}$ & $\begin{array}{l}\text { Penalty due to being infected } \\
\text { (e.g. disease complications) }\end{array}$ & 0.3 \\
\hline$L$ & Baseline payoff & 1 \\
\hline$P_{V}$ & $\begin{array}{l}\text { Payoff to vaccinate as soon as } \\
\text { index case exhibits symptoms }\end{array}$ & \\
\hline$P_{N}$ & Payoff not to vaccinate & \\
\hline
\end{tabular}

Due to the correction in the payoff to vaccinate, a new set of parameter values were used and are listed in Table 1. The overall conclusion of the model did not change, with the Figures being very similar to previous. The only notable change was in the sensitivity analysis. The distribution of secondary infections and vaccinators no longer remains bimodal for a range of values for the probability of transmission, $p$ (Figs. 1, 2). The distribution of secondary infections and vaccinators does remain bimodal for a small range of values for vaccine efficacy, $\varepsilon$ (Figs. 1, 2). 

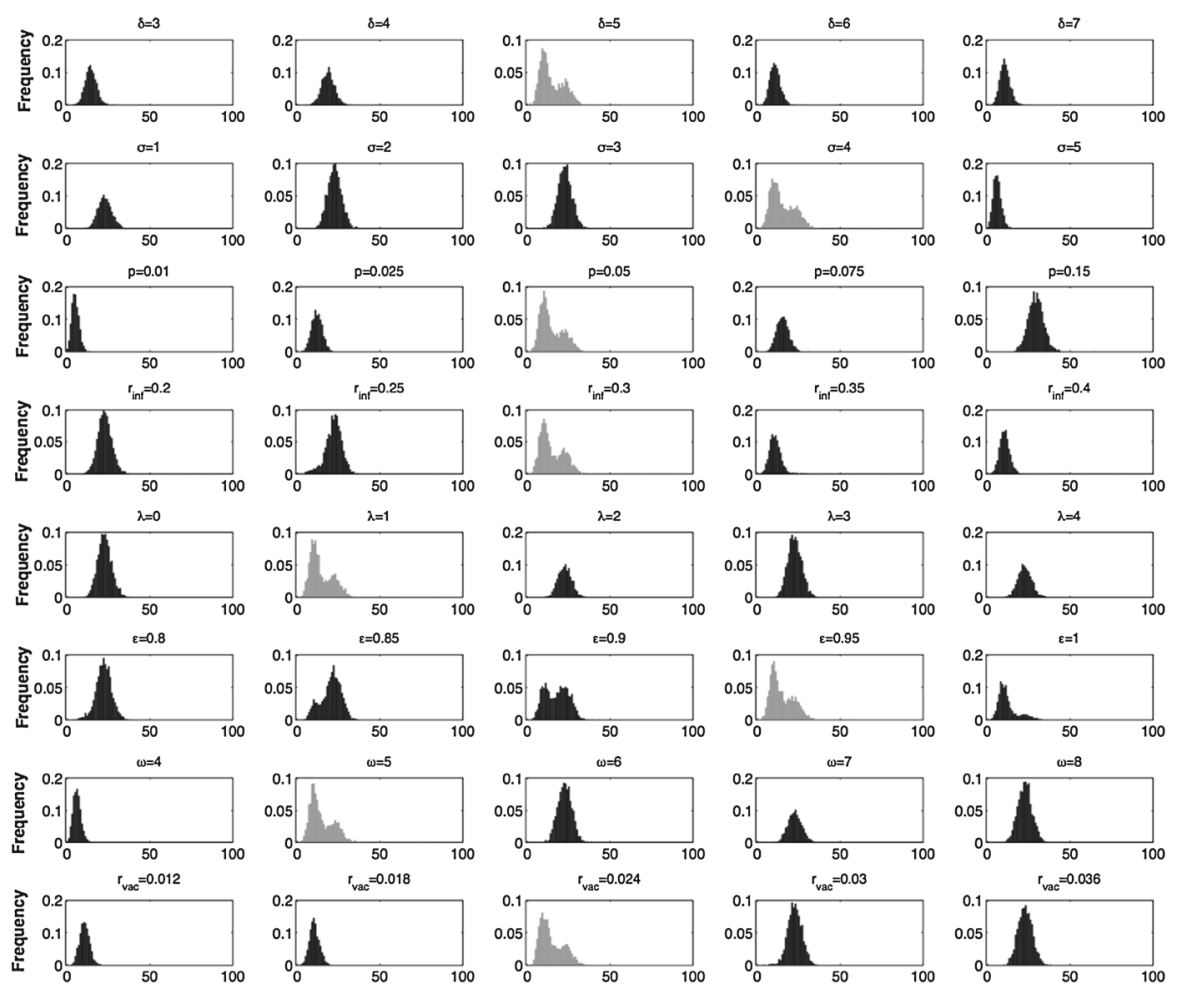

Fig. 1 The distribution of the infected individuals under full imitation for the case of $Q=100$ for the parameters $\lambda, \varepsilon, \omega, r_{\mathrm{vac}}, r_{\mathrm{inf}}, p, \sigma$, and $\delta$. The baseline parameter values are used to generate the gray distributions and black distributions represent variations from the baseline 

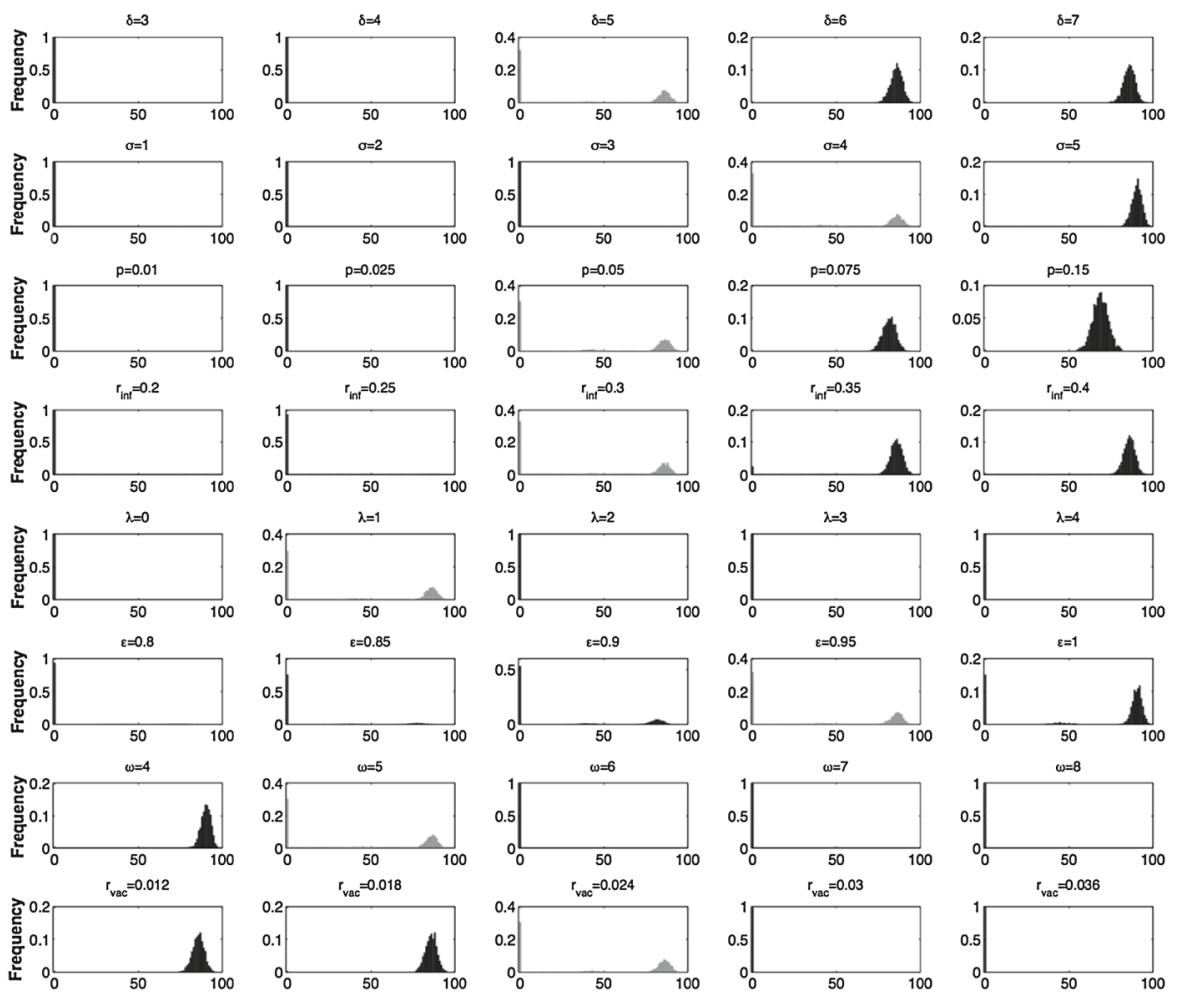

Fig. 2 The distribution of the vaccinated under full imitation for the case of $Q=100$ for the parameters $\lambda, \varepsilon, \omega, r_{\mathrm{vac}}, r_{\mathrm{inf}}, p, \sigma$, and $\delta$. The baseline parameter values are used to generate the gray distributions and black distributions represent variations from the baseline 